\title{
Factors Affecting the Design and Economic Operation of Distribution Networks and Its Investigation in the 0.4 KV Distribution Network of Ghazni City
}

\section{Massoud Danishmal}

Electrical Power Engineering Department, Ghazni Technical University, Afghanistan

\section{Email: Massoudzeyarmal@gmail.com}

Phone: +93782586653

\begin{abstract}
:
The design of power distribution systems should be such that it can technically respond to the increase in electricity demand properly and economically, optimally designed and high network reliability. In order to respond to the increase in electricity demand, load forecasting must be done so that in addition to providing the electricity needed by customers, expansion of power generation centers, expansion of substations, expansion of transformer stations and selection of their appropriate location can be done optimally. In this article, we first examine the definitions and factors that are technically and economically effective in the economic design of energy distribution systems. And in the next stage, we will see whether these abovementioned effective factors are considered in the $0.4 \mathrm{kV}$ distribution network of Ghazni city or not.
\end{abstract}

Key word: Electricity demand, Transformer station, Distribution systems, Design of Energy Distribution Systems, Subscribers 


\section{Introduction}

Ghazni city for the first time in 04/01/2018 benefited from imported electricity. A part of Ghazni city has been supplied with electricity by a diesel generator and private companies since 1977 using a non-standard network. Currently, about 4.5 megawatts of electricity is distributed in Ghazni city and it is planned to increase to 25 megawatts in the coming years. The distribution network of Ghazni city in the fourth period of 1398 had $11.66 \%$ of waste and the lowest price of peak voltage was in the last part of Pashtunabad village, which reached 180 volts. Medium voltage networks (20 kV and less), low voltage and distribution systems are occasionally developed to be able to power new consumers.

If this development is not planned according to international standards and standards, and this is accompanied by poor quality equipment, damaged buildings and insufficient maintenance and care, as well as theft of energy, defects in the meter system, electricity transmitted without meters, etc. It will result in an extraordinary loss of electricity and low quality standards and reliability of extremely low consumption in the systems. As a result, the transmission of electrical energy by the power grid system from the source to the consumer will waste the main equipment (line and transformer), will cause a certain amount of electrical energy to be absorbed by the active resistors of the equipment and transferred to the environment. The second compound is passive resistors that convert electrical energy into electromagnetic energy.

\section{Research Methodology}

This scientific article is a field research article. The present research method is divided into two main parts:

1- Gathering information

2- Examining the samples and analyzing the obtained knowledge.

Data collection has been done in two ways:

Observation method and documentary method 
Factors affecting the network design and economic operation of electrical distribution systems

One of the main tasks of power distribution companies is to provide constant voltage and frequency for subscribers, continuity of power supply and safety of the distribution network. The selection of electricity distribution network is done according to factors such as route (lane length, route type, and transverse route limitation), population density, economic factors and environmental beauty. Unauthorized use of $0.4 \mathrm{kV}$ distribution networks is easier and more common due to the availability of the network, and preventing it, in addition to having a cost, causes local social tensions.

In general, the following points should be considered for the design of the distribution network:

- Full knowledge of the geographical location of the place

- Prepare location maps to standard scales

- Determine the number of subscribers by their energy consumption and their distance from the power transformer.

- Determining the cross section of network wires by considering the power consumption on each circuit and their distance from the power transformer.

- Place the transformers in the center of gravity of the load (in this case the voltage loss is minimized)

- The length of each circuit of the transformer should not exceed the allowable limit (500600) meters.

- The possibility of future development and increase in consumption should be considered.

The amount of load consumed should not be considered as the maximum total load, but the load synchronization coefficient should be calculated by considering the load factor and the required transformer should be selected. Determining the capacity of the transformer using existing international standards.

Considering that the electricity industry is one of the most vital industries in the country, so it requires great attention and investment, 
This industry needs huge and long-term investment in production, transmission and distribution, which is part of the country's economic potential Focuses on itself.

Current distribution networks have many problems in proper performance. These problems create difficult conditions for operation, especially in periods of maximum demand, and reduce the quality and reliability of the system.

\section{Electric load graph}

Load refers to a device or set of devices or consumers that receives electricity from the grid. Electric load graph is a graph of consumption changes in time, which is shown daily, weekly, monthly and annually. Load forecast of a city or region Electric load graph is usually drawn monthly, seasonally and annually. Different consumers do not receive their maximum load simultaneously from the network and depending on the type of consumption, the time to receive the maximum varies. Figure (1) shows the electric charge graph of the Ghazni Breshna distribution network in 24 hours

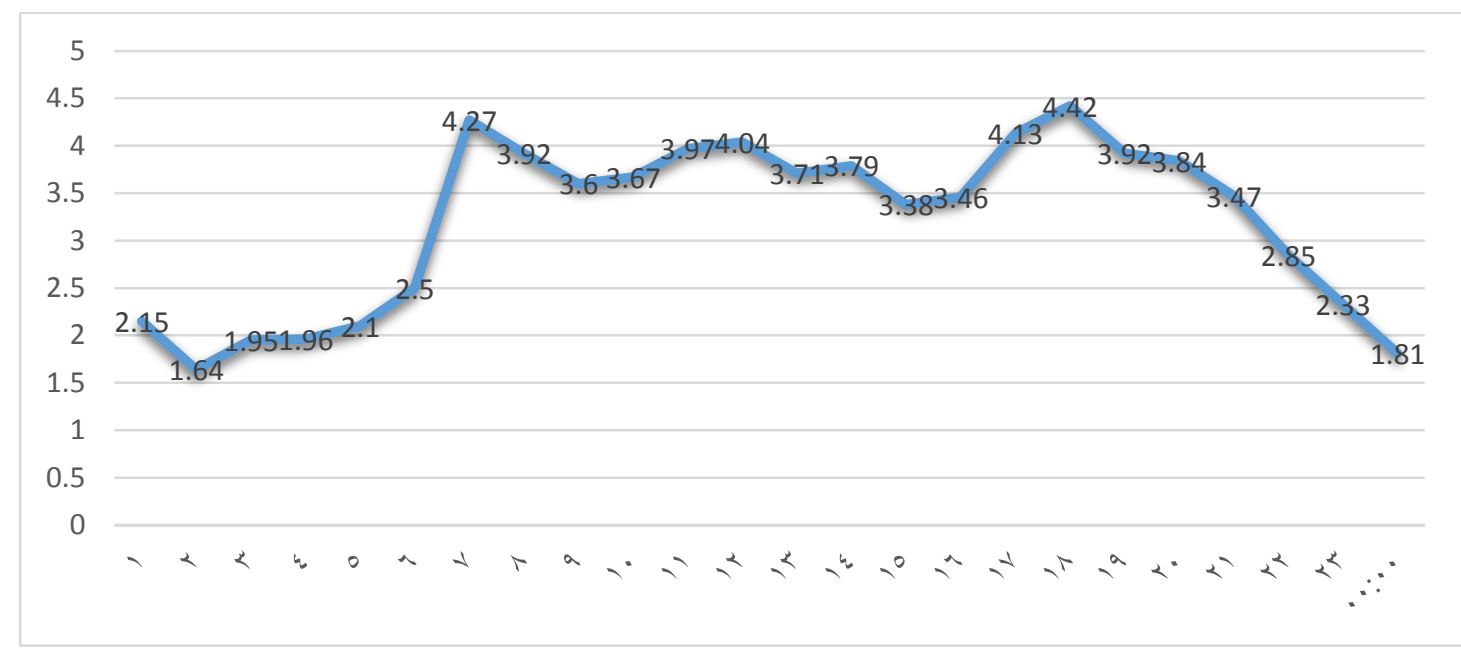

Electric load graph of Ghazni distribution network in 24 hours.

With the electric charge graph, we can obtain the average load and the load factor.

$m=\frac{P_{m}}{P_{\max }}=\frac{3.203}{4.42}=0.724$ 
The closer the load factor is to one, the optimal use of the networks and the smoother the load graph, ie the average load is closer to the maximum load, which is very low in the distribution network of Ghazni city.

\section{Type of load}

In selecting the network, the choice of load type (residential, commercial, industrial, peasant loads) must be specified Factors influencing overload, which are (population, civilization and improving living standards) must be considered. Factors influencing load forecasting are:

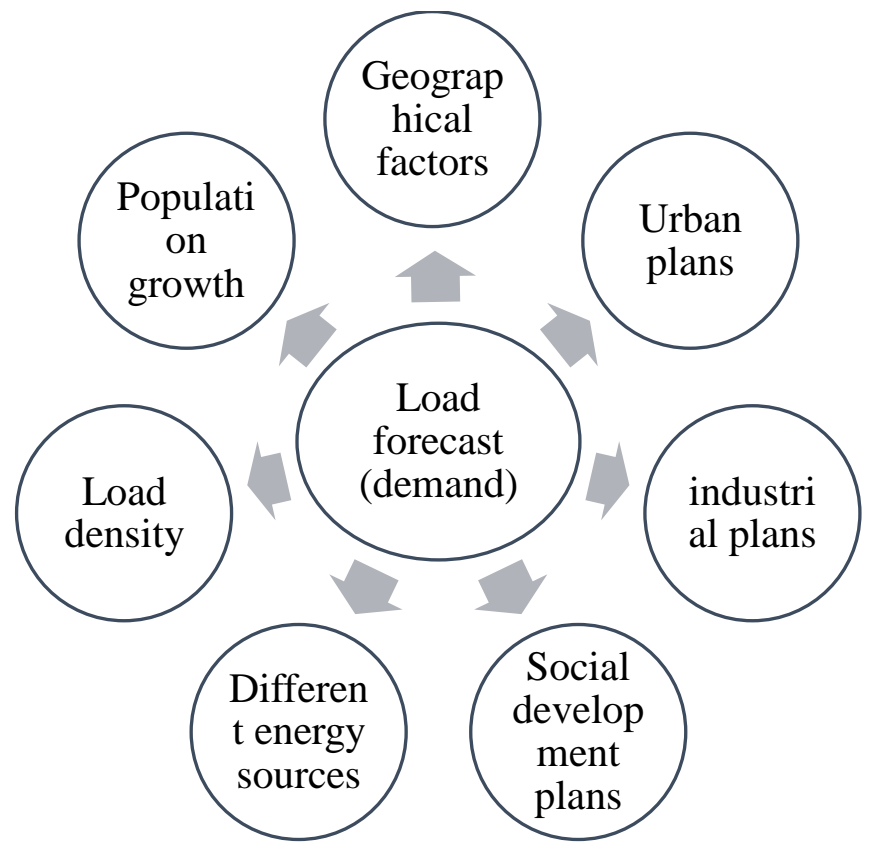

Factors affecting load forecasting

Considering the synchronization coefficient in the electricity distribution network is one of the effective factors.

Considering that the satisfaction of the subscribers is finally tangible in the distribution sector and the smallest defect shows itself more. Therefore, in this regard, serious measures in power distribution networks for the design and optimal use of power systems and compliance with standards is a necessity. Due to the vastness of electricity distribution networks on the one hand and the lack of engineering of these networks due to its high age, it is necessary to review these networks. 
Academic Journal of Research and Scientific Publishing | Vol 3 | Issue 27

Publication Date: 5-7-2021 ISSN: 2706-6495

In the table below, the state of $0.4 \mathrm{kV}$ distribution network in Ghazni city is briefly studied.

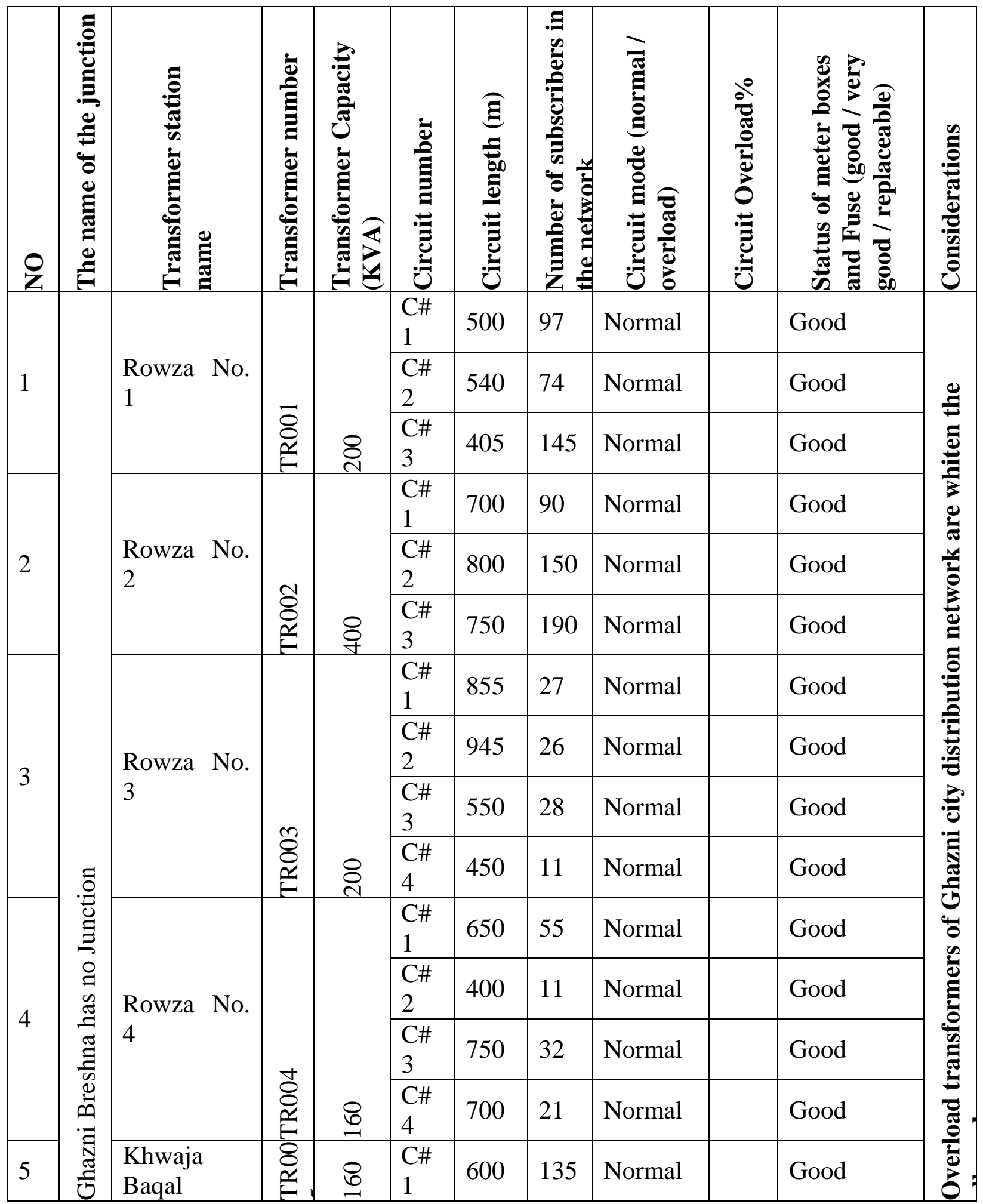


Academic Journal of Research and Scientific Publishing | Vol 3 | Issue 27

Publication Date: 5-7-2021 ISSN: 2706-6495

\begin{tabular}{|c|c|c|c|c|c|c|c|c|c|}
\hline & & & & $\begin{array}{l}\text { C\# } \\
2 \\
\end{array}$ & 500 & 120 & Normal & & Good \\
\hline \multirow{4}{*}{6} & \multirow{4}{*}{$\begin{array}{l}\text { Shahre } \\
\text { Kohna }\end{array}$} & \multirow[b]{4}{*}{ 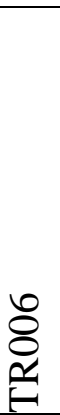 } & \multirow[b]{4}{*}{ \& } & $\begin{array}{l}\mathrm{C \#} \\
1\end{array}$ & 730 & 122 & $\begin{array}{l}\text { Overloa } \\
\text { d }\end{array}$ & $\begin{array}{l}15 \\
\%\end{array}$ & $\begin{array}{l}\text { Replaceabl } \\
\mathrm{e}\end{array}$ \\
\hline & & & & $\begin{array}{l}\mathrm{C \#} \\
2\end{array}$ & 758 & 141 & Normal & & $\begin{array}{l}\text { Replaceabl } \\
\text { e }\end{array}$ \\
\hline & & & & $\begin{array}{l}\mathrm{C \#} \\
3\end{array}$ & 464 & 156 & Normal & & $\begin{array}{l}\text { Replaceabl } \\
\text { e }\end{array}$ \\
\hline & & & & $\begin{array}{l}\text { C\# } \\
4\end{array}$ & 435 & 189 & $\begin{array}{l}\text { Overloa } \\
\mathrm{d}\end{array}$ & $\begin{array}{l}20 \\
\%\end{array}$ & $\begin{array}{l}\text { Replaceabl } \\
\text { e }\end{array}$ \\
\hline \multirow{3}{*}{7} & \multirow{3}{*}{$\begin{array}{l}\text { Bahlool } \\
\text { Sahib }\end{array}$} & \multirow[b]{3}{*}{$\begin{array}{l}5 \\
8 \\
\frac{8}{6}\end{array}$} & \multirow[b]{3}{*}{8} & $\begin{array}{l}\text { C\# } \\
1\end{array}$ & 630 & 108 & Normal & & $\begin{array}{l}\text { Replaceabl } \\
\text { e }\end{array}$ \\
\hline & & & & $\begin{array}{l}\text { C\# } \\
2 \\
\end{array}$ & 350 & 66 & Normal & & Good \\
\hline & & & & $\begin{array}{l}\text { C\# } \\
3 \\
\end{array}$ & 495 & 88 & Normal & & $\begin{array}{l}\text { Replaceabl } \\
\mathrm{e}\end{array}$ \\
\hline \multirow{3}{*}{8} & \multirow{3}{*}{$\begin{array}{l}\text { Al-Biruni } \\
\text { (Bahlool } \\
\text { Saheb } \\
\text { Gardens) }\end{array}$} & \multirow[b]{3}{*}{ 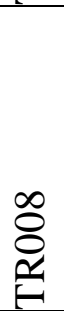 } & \multirow[b]{3}{*}{8} & $\begin{array}{l}\mathrm{C \#} \\
1 \\
\end{array}$ & 808 & 102 & Normal & & Good \\
\hline & & & & $\begin{array}{l}\mathrm{C \#} \\
2 \\
\end{array}$ & 612 & 149 & Normal & & Good \\
\hline & & & & $\begin{array}{l}\text { C\# } \\
3\end{array}$ & 941 & 17 & Normal & & Good \\
\hline \multirow{6}{*}{9} & \multirow{6}{*}{$\begin{array}{l}\text { Diesel } \\
\text { House No. } 1\end{array}$} & \multirow[b]{6}{*}{$\begin{array}{l}8 \\
8 \\
\text { dx }\end{array}$} & \multirow[b]{6}{*}{8} & $\begin{array}{l}\text { C\# } \\
1 \\
\end{array}$ & $\begin{array}{l}126 \\
0\end{array}$ & 173 & $\begin{array}{l}\text { Overloa } \\
\mathrm{d}\end{array}$ & $\begin{array}{l}20 \\
\%\end{array}$ & $\begin{array}{l}\text { Replaceabl } \\
\mathrm{e}\end{array}$ \\
\hline & & & & $\begin{array}{l}\text { C\# } \\
2\end{array}$ & 900 & 111 & Normal & & $\begin{array}{l}\text { Replaceabl } \\
\text { e }\end{array}$ \\
\hline & & & & $\begin{array}{l}\mathrm{C \#} \\
3 \\
\end{array}$ & 700 & 3 & $\begin{array}{l}\text { Overloa } \\
\mathrm{d}\end{array}$ & $\begin{array}{l}15 \\
\%\end{array}$ & $\begin{array}{l}\text { Replaceabl } \\
\mathrm{e}\end{array}$ \\
\hline & & & & $\begin{array}{l}\text { C\# } \\
4 \\
\end{array}$ & 900 & 156 & Normal & & $\begin{array}{l}\text { Replaceabl } \\
\text { e }\end{array}$ \\
\hline & & & & $\begin{array}{l}\mathrm{C \#} \\
5\end{array}$ & 900 & 90 & Normal & & $\begin{array}{l}\text { Replaceabl } \\
\text { e }\end{array}$ \\
\hline & & & & $\begin{array}{l}\text { C\# } \\
6 \\
\end{array}$ & $\begin{array}{l}115 \\
0\end{array}$ & 137 & Normal & & $\begin{array}{l}\text { Replaceabl } \\
\mathrm{e}\end{array}$ \\
\hline \multirow{3}{*}{10} & \multirow{3}{*}{$\begin{array}{l}\text { Hakim } \\
\text { Sahib No. } 1\end{array}$} & \multirow{3}{*}{$\begin{array}{l}\stackrel{0}{\circ} \\
\stackrel{2}{\mathscr{v}}\end{array}$} & \multirow[b]{3}{*}{$\stackrel{\circ}{2}$} & $\begin{array}{l}\mathrm{C \#} \\
1\end{array}$ & 600 & 42 & Normal & & Good \\
\hline & & & & $\begin{array}{l}\text { C\# } \\
2 \\
\end{array}$ & 500 & 29 & Normal & & Good \\
\hline & & & & $\begin{array}{l}\text { C\# } \\
3 \\
\end{array}$ & 550 & 20 & Normal & & Good \\
\hline 11 & $\begin{array}{l}\text { Hakim } \\
\text { Saheb No. } 2\end{array}$ & $\begin{array}{l}\overline{0} \\
\bar{f}\end{array}$ & \&్ర & $\begin{array}{l}\mathrm{C} \# \\
1 \\
\end{array}$ & 228 & 76 & Normal & & Good \\
\hline
\end{tabular}


Academic Journal of Research and Scientific Publishing | Vol 3 | Issue 27

Publication Date: 5-7-2021 ISSN: 2706-6495

\begin{tabular}{|c|c|c|c|c|c|c|c|c|c|}
\hline & & & & $\begin{array}{l}\text { C\# } \\
2\end{array}$ & 284 & 51 & Normal & & Good \\
\hline & & & & $\begin{array}{l}\text { C\# } \\
3\end{array}$ & 389 & 47 & Normal & & Good \\
\hline \multirow{3}{*}{12} & \multirow{3}{*}{$\begin{array}{l}\text { Shamir } \\
\text { Sahib }\end{array}$} & \multirow[b]{3}{*}{$\begin{array}{l}\stackrel{N}{0} \\
\frac{1}{21}\end{array}$} & \multirow[b]{3}{*}{8} & $\begin{array}{l}\text { C\# } \\
1 \\
\end{array}$ & 581 & 90 & Normal & & $\begin{array}{l}\text { Replaceabl } \\
\text { e }\end{array}$ \\
\hline & & & & $\begin{array}{l}\text { C\# } \\
2 \\
\end{array}$ & 730 & 213 & $\begin{array}{l}\text { Overloa } \\
\mathrm{d}\end{array}$ & $\begin{array}{l}20 \\
\%\end{array}$ & $\begin{array}{l}\text { Replaceabl } \\
\mathrm{e}\end{array}$ \\
\hline & & & & $\begin{array}{l}\text { C\# } \\
3 \\
\end{array}$ & 419 & 122 & Normal & & $\begin{array}{l}\text { Replaceabl } \\
\mathrm{e}\end{array}$ \\
\hline \multirow{3}{*}{13} & \multirow{3}{*}{$\begin{array}{l}\text { Qala-e- } \\
\text { Now sare } \\
\text { reg }\end{array}$} & \multirow[b]{3}{*}{$\frac{m}{2}$} & \multirow[b]{3}{*}{ శ్ } & $\begin{array}{l}\text { C\# } \\
1 \\
\end{array}$ & 250 & 34 & Normal & & Good \\
\hline & & & & $\begin{array}{l}\text { C\# } \\
2\end{array}$ & 650 & 153 & Normal & & Good \\
\hline & & & & $\begin{array}{l}\text { C\# } \\
3 \\
\end{array}$ & 600 & 66 & Normal & & Good \\
\hline \multirow{5}{*}{14} & \multirow{5}{*}{$\begin{array}{l}\text { Diesel } \\
\text { House No. } 2\end{array}$} & \multirow[b]{5}{*}{$\frac{⿱}{2}$} & \multirow[b]{5}{*}{8} & $\begin{array}{l}\text { C\# } \\
1 \\
\end{array}$ & $\begin{array}{l}108 \\
0 \\
\end{array}$ & 138 & Normal & & Good \\
\hline & & & & $\begin{array}{l}\text { C\# } \\
2\end{array}$ & 990 & 315 & Normal & & Good \\
\hline & & & & $\begin{array}{l}\text { C\# } \\
3 \\
\end{array}$ & 300 & 39 & Normal & & Good \\
\hline & & & & $\begin{array}{l}\text { C\# } \\
4\end{array}$ & 350 & 11 & Normal & & $\begin{array}{l}\text { Replaceabl } \\
\mathrm{e}\end{array}$ \\
\hline & & & & $\begin{array}{l}\text { C\# } \\
5 \\
\end{array}$ & 855 & 35 & Normal & & Good \\
\hline \multirow{3}{*}{15} & \multirow{3}{*}{$\begin{array}{l}\text { Qala-e- } \\
\text { Now } \\
\text { Khajeh } \\
\text { Roshnai }\end{array}$} & \multirow[b]{3}{*}{$\begin{array}{l}\frac{n}{o} \\
\stackrel{1}{H}\end{array}$} & \multirow[b]{3}{*}{$\underset{8}{0}$} & $\begin{array}{l}\text { C\# } \\
1 \\
\end{array}$ & 415 & 75 & Normal & & Good \\
\hline & & & & $\begin{array}{l}\text { C\# } \\
2\end{array}$ & 743 & 144 & Normal & & Good \\
\hline & & & & $\begin{array}{l}\text { C\# } \\
3 \\
\end{array}$ & 994 & 122 & Normal & & Good \\
\hline \multirow{3}{*}{16} & \multirow{3}{*}{$\begin{array}{l}\text { Maihan } \\
\text { Abad }\end{array}$} & \multirow{3}{*}{ 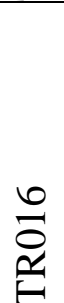 } & \multirow[b]{3}{*}{ 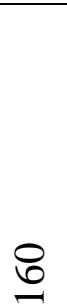 } & $\begin{array}{l}\text { C\# } \\
1\end{array}$ & 265 & 24 & Normal & & Good \\
\hline & & & & $\begin{array}{l}\text { C\# } \\
2 \\
\end{array}$ & 300 & 52 & Normal & & Good \\
\hline & & & & $\begin{array}{l}\text { C\# } \\
3\end{array}$ & 87 & 4 & Normal & & Good \\
\hline \multirow{2}{*}{17} & \multirow{2}{*}{$\begin{array}{l}\text { Pashtun } \\
\text { Abad No. } 1\end{array}$} & \multirow{2}{*}{$\frac{0}{2}$} & \multirow[b]{2}{*}{8} & $\begin{array}{l}\text { C\# } \\
1 \\
\end{array}$ & 491 & 59 & Normal & & Good \\
\hline & & & & $\begin{array}{l}\text { C\# } \\
2\end{array}$ & 148 & 9 & Normal & & Good \\
\hline
\end{tabular}


Academic Journal of Research and Scientific Publishing | Vol 3 | Issue 27

Publication Date: 5-7-2021 ISSN: 2706-6495

\begin{tabular}{|c|c|c|c|c|c|c|c|c|c|}
\hline & & & & $\begin{array}{l}\mathrm{C} \# \\
3\end{array}$ & 678 & 158 & Normal & & $\begin{array}{l}\text { Replaceabl } \\
\text { e }\end{array}$ \\
\hline & & & & $\begin{array}{l}\text { C\# } \\
4\end{array}$ & 495 & 43 & Normal & & Good \\
\hline & & & & $\begin{array}{l}\mathrm{C} \# \\
1\end{array}$ & 552 & 99 & Normal & & Good \\
\hline 18 & $\begin{array}{l}\text { Pashtun } \\
\text { Abad No. } 2\end{array}$ & & & $\begin{array}{l}\text { C\# } \\
2\end{array}$ & 414 & 90 & Normal & & Good \\
\hline & & 定 & \&్ర & $\begin{array}{l}\mathrm{C} \# \\
3 \\
\end{array}$ & 475 & 64 & Normal & & $\begin{array}{l}\text { Replaceabl } \\
\mathrm{e}\end{array}$ \\
\hline & Qala-e- & & & $\begin{array}{l}\mathrm{C} \# \\
1\end{array}$ & 484 & 60 & Normal & & Good \\
\hline 19 & $\begin{array}{l}\text { Amir } \\
\text { Mohammad }\end{array}$ & & & $\begin{array}{l}\mathrm{C} \# \\
2\end{array}$ & 400 & 106 & $\begin{array}{l}\text { Overloa } \\
\mathrm{d}\end{array}$ & $\begin{array}{l}20 \\
\%\end{array}$ & $\begin{array}{l}\text { Replaceabl } \\
\text { e }\end{array}$ \\
\hline & Khan No. 1 & 定 & $\stackrel{8}{8}$ & $\begin{array}{l}\mathrm{C} \# \\
3 \\
\end{array}$ & 921 & 132 & Normal & & Good \\
\hline & Qala-e- & & & $\begin{array}{l}\mathrm{C} \# \\
1\end{array}$ & 945 & 155 & Normal & & Good \\
\hline 20 & $\begin{array}{l}\text { Amir } \\
\text { Mohammad }\end{array}$ & & & $\begin{array}{l}\text { C\# } \\
2\end{array}$ & 150 & 17 & Normal & & Good \\
\hline & Khan No. 2 & 客 & \& & $\begin{array}{l}\text { C\# } \\
3\end{array}$ & 498 & 49 & $\begin{array}{l}\text { Overloa } \\
\mathrm{d}\end{array}$ & $\begin{array}{l}10 \\
\%\end{array}$ & $\begin{array}{l}\text { Replaceabl } \\
\mathrm{e}\end{array}$ \\
\hline & & & & $\begin{array}{l}\text { C\# } \\
1 \\
\end{array}$ & 810 & 119 & Normal & & $\begin{array}{l}\text { Replaceabl } \\
\text { e }\end{array}$ \\
\hline & & & & $\begin{array}{l}\mathrm{C} \# \\
2\end{array}$ & 855 & 149 & Normal & & Good \\
\hline 21 & Fourth Plan & & & $\begin{array}{l}\mathrm{C} \# \\
3 \\
\end{array}$ & 585 & 69 & Normal & & Good \\
\hline & & $\begin{array}{l}\overrightarrow{\widehat{\Omega}} \\
\text { s. } \\
\end{array}$ & $\bigotimes_{\infty}$ & $\begin{array}{l}\mathrm{C} \# \\
4 \\
\end{array}$ & 810 & 97 & Normal & & Good \\
\hline 22 & $\begin{array}{l}\text { Saqafat } \\
\text { center }\end{array}$ & 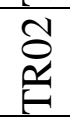 & in & \begin{tabular}{|l}
$\mathrm{C} \#$ \\
1 \\
\end{tabular} & 400 & 1 & Normal & & Good \\
\hline & Faiz & & & $\begin{array}{l}\mathrm{C} \# \\
1 \\
\end{array}$ & 300 & 119 & Normal & & $\begin{array}{l}\text { Replaceabl } \\
\mathrm{e}\end{array}$ \\
\hline 23 & $\begin{array}{l}\text { Mohammad } \\
\text { Katib Road }\end{array}$ & & & $\begin{array}{l}\mathrm{C} \# \\
2\end{array}$ & 450 & 123 & Normal & & Good \\
\hline & No. 1 & 客 & 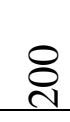 & $\begin{array}{l}\text { C\# } \\
3 \\
\end{array}$ & 250 & 31 & Normal & & Good \\
\hline 24 & $\begin{array}{l}\text { faiz } \\
\text { Mohammad }\end{array}$ & & & $\begin{array}{l}\mathrm{C} \# \\
1\end{array}$ & 850 & 92 & Normal & & Good \\
\hline 24 & $\begin{array}{l}\text { Katib Road } \\
\text { No. } 2\end{array}$ & है & 8 & $\begin{array}{l}\text { C\# } \\
2\end{array}$ & 700 & 107 & Normal & & $\begin{array}{l}\text { Replaceabl } \\
\mathrm{e}\end{array}$ \\
\hline
\end{tabular}


Academic Journal of Research and Scientific Publishing | Vol 3 | Issue 27

Publication Date: 5-7-2021 ISSN: 2706-6495

\begin{tabular}{|c|c|c|c|c|c|c|c|c|}
\hline & & & & $\begin{array}{l}\text { C\# } \\
3 \\
\end{array}$ & 550 & 134 & Normal & Good \\
\hline & & & & $\begin{array}{l}\text { C\# } \\
4 \\
\end{array}$ & 500 & 84 & Normal & Good \\
\hline 25 & $\begin{array}{l}\text { Nawabad } \\
\text { Oadam }\end{array}$ & & & $\begin{array}{l}\text { C\# } \\
1\end{array}$ & 550 & 147 & Normal & Good \\
\hline $2 J$ & Khan Castle & ำ & $\stackrel{\circ}{2}$ & $\begin{array}{l}\text { C\# } \\
2 \\
\end{array}$ & 600 & 115 & Normal & Good \\
\hline & Qadam & & & $\begin{array}{l}\text { C\# } \\
1\end{array}$ & 900 & 104 & Normal & Good \\
\hline 20 & $\begin{array}{l}\text { Khan Castle } \\
\text { Hill }\end{array}$ & $\begin{array}{l}\text { ป } \\
\text { ô } \\
\text { E }\end{array}$ & 尺̊ำ & $\begin{array}{l}\text { C\# } \\
2 \\
\end{array}$ & 450 & 147 & Normal & Good \\
\hline & & & & $\begin{array}{l}\mathrm{C \#} \\
1 \\
\end{array}$ & 400 & 26 & Normal & Good \\
\hline & Qadam & & & $\begin{array}{l}\text { C\# } \\
2 \\
\end{array}$ & 800 & 92 & Normal & Good \\
\hline 21 & Khan Castle & & & $\begin{array}{l}\text { C\# } \\
3 \\
\end{array}$ & 500 & 20 & Normal & Good \\
\hline & & 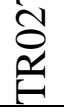 & §̊ & $\begin{array}{l}\text { C\# } \\
4 \\
\end{array}$ & 250 & 45 & Normal & Good \\
\hline & & & & $\begin{array}{l}\mathrm{C \#} \\
1 \\
\end{array}$ & 600 & 122 & Normal & $\begin{array}{l}\text { Replaceabl } \\
\text { e }\end{array}$ \\
\hline & Haider & & & $\begin{array}{l}\text { C\# } \\
2 \\
\end{array}$ & 450 & 191 & Normal & $\begin{array}{l}\text { Replaceabl } \\
\mathrm{e}\end{array}$ \\
\hline 28 & Abad No. 1 & & & $\begin{array}{l}\text { C\# } \\
3\end{array}$ & 500 & 69 & Normal & $\begin{array}{l}\text { Replaceabl } \\
\text { e }\end{array}$ \\
\hline & & तิ & ஓ्र & $\begin{array}{l}\text { C\# } \\
4 \\
\end{array}$ & 300 & 54 & Normal & Good \\
\hline & & & & $\begin{array}{l}\text { C\# } \\
1 \\
\end{array}$ & 300 & 160 & Normal & $\begin{array}{l}\text { Replaceabl } \\
\mathrm{e}\end{array}$ \\
\hline & Haider & & & $\begin{array}{l}\text { C\# } \\
2 \\
\end{array}$ & 750 & 138 & Normal & $\begin{array}{l}\text { Replaceabl } \\
\text { e }\end{array}$ \\
\hline 29 & Abad No. 2 & & & $\begin{array}{l}\text { C\# } \\
3\end{array}$ & 650 & 40 & Normal & Good \\
\hline & & ญิ & ஓ & $\begin{array}{l}\text { C\# } \\
4\end{array}$ & 350 & 11 & Normal & Good \\
\hline & & & & $\begin{array}{l}\text { C\# } \\
1 \\
\end{array}$ & 765 & 44 & Normal & Good \\
\hline 30 & Sanjetak & & & $\begin{array}{l}\text { C\# } \\
2\end{array}$ & 500 & 23 & Normal & Good \\
\hline & & ô & $\underset{8}{8}$ & $\begin{array}{l}\text { C\# } \\
3 \\
\end{array}$ & $\begin{array}{l}120 \\
0\end{array}$ & 74 & Normal & Good \\
\hline
\end{tabular}


Academic Journal of Research and Scientific Publishing | Vol 3 | Issue 27

Publication Date: 5-7-2021 ISSN: 2706-6495

\begin{tabular}{|c|c|c|c|c|c|c|c|c|c|}
\hline \multirow{2}{*}{31} & \multirow{2}{*}{$\begin{array}{l}\text { Khwaja Ali } \\
\text { Sahib }\end{array}$} & \multirow[b]{2}{*}{ 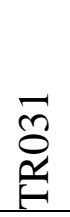 } & \multirow[b]{2}{*}{ ฉ̊ } & $\begin{array}{l}\mathrm{C} \# \\
1\end{array}$ & 550 & 205 & Normal & & Good \\
\hline & & & & $\begin{array}{l}\text { C\# } \\
2\end{array}$ & 850 & 86 & Normal & & Good \\
\hline \multirow{4}{*}{32} & \multirow{4}{*}{ Third Plan } & \multirow[b]{4}{*}{ 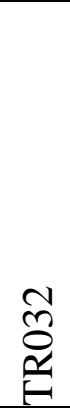 } & \multirow[b]{4}{*}{$\underset{\infty}{\infty}$} & $\begin{array}{l}\text { C\# } \\
1\end{array}$ & 990 & 99 & Normal & & Good \\
\hline & & & & $\begin{array}{l}\mathrm{C \#} \\
2\end{array}$ & 810 & 110 & Normal & & Good \\
\hline & & & & $\begin{array}{l}\mathrm{C \#} \\
3\end{array}$ & 675 & 61 & Normal & & Good \\
\hline & & & & $\begin{array}{l}\text { C\# } \\
4\end{array}$ & 250 & 19 & Normal & & Good \\
\hline \multirow{4}{*}{33} & \multirow{4}{*}{$\begin{array}{l}\text { Said Ahmad } \\
\text { Makaee }\end{array}$} & \multirow[b]{4}{*}{$\begin{array}{l}\text { } \\
\hat{\varepsilon}\end{array}$} & \multirow[b]{4}{*}{$\delta_{\infty}$} & $\begin{array}{l}\text { C\# } \\
1\end{array}$ & 550 & 99 & Normal & & Good \\
\hline & & & & $\begin{array}{l}\mathrm{C} \# \\
2\end{array}$ & 850 & 67 & Normal & & $\begin{array}{l}\text { Replaceabl } \\
\mathrm{e}\end{array}$ \\
\hline & & & & $\begin{array}{l}\text { C\# } \\
3\end{array}$ & 650 & 57 & Normal & & Good \\
\hline & & & & $\begin{array}{l}\mathrm{C} \# \\
4 \\
\end{array}$ & 550 & 135 & $\begin{array}{l}\text { Overloa } \\
\mathrm{d}\end{array}$ & $\begin{array}{l}25 \\
\% \\
\end{array}$ & $\begin{array}{l}\text { Replaceabl } \\
\mathrm{e}\end{array}$ \\
\hline \multirow{4}{*}{34} & \multirow{4}{*}{$\begin{array}{l}\text { Qala-e- } \\
\text { Tahwildara } \\
\mathrm{n}\end{array}$} & \multirow[b]{4}{*}{ 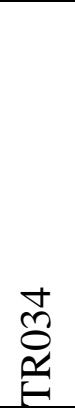 } & \multirow[b]{4}{*}{ ถู } & $\begin{array}{l}\mathrm{C} \# \\
1\end{array}$ & 850 & 183 & Normal & & Good \\
\hline & & & & $\begin{array}{l}\mathrm{C \#} \\
2\end{array}$ & 350 & 99 & Normal & & Good \\
\hline & & & & $\begin{array}{l}\text { C\# } \\
3\end{array}$ & 400 & 25 & Normal & & Good \\
\hline & & & & $\begin{array}{l}\text { C\# } \\
4\end{array}$ & $\begin{array}{l}100 \\
0\end{array}$ & 130 & Normal & & Good \\
\hline \multirow{4}{*}{35} & \multirow{4}{*}{$\begin{array}{l}\text { Qala-e- } \\
\text { Ahengran }\end{array}$} & \multirow{4}{*}{ 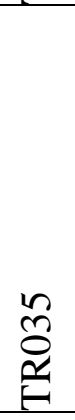 } & \multirow[b]{4}{*}{ 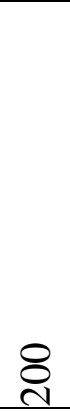 } & $\begin{array}{l}\text { C\# } \\
1\end{array}$ & 700 & 57 & Normal & & Good \\
\hline & & & & $\begin{array}{l}\text { C\# } \\
2\end{array}$ & 500 & 48 & Normal & & Good \\
\hline & & & & $\begin{array}{l}\mathrm{C} \# \\
3\end{array}$ & 495 & 68 & Normal & & Good \\
\hline & & & & $\begin{array}{l}\mathrm{C} \# \\
4\end{array}$ & 540 & 35 & Normal & & Good \\
\hline \multirow{3}{*}{36} & \multirow{3}{*}{$\begin{array}{l}\text { Khashik } \\
\text { No. } 1\end{array}$} & \multirow{3}{*}{ 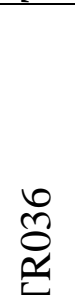 } & \multirow[b]{3}{*}{8} & $\begin{array}{l}\mathrm{C} \# \\
1\end{array}$ & 855 & 156 & Normal & & Good \\
\hline & & & & $\begin{array}{l}\text { C\# } \\
2\end{array}$ & 400 & 94 & Normal & & Good \\
\hline & & & & $\begin{array}{l}\text { C\# } \\
3\end{array}$ & 720 & 39 & Normal & & Good \\
\hline
\end{tabular}




\begin{tabular}{|c|c|c|c|c|c|c|c|c|}
\hline \multirow{4}{*}{37} & \multirow{4}{*}{$\begin{array}{l}\text { Khashik } \\
\text { No. } 2\end{array}$} & \multirow[b]{4}{*}{$\begin{array}{l}\hat{n} \\
0 \\
\hat{\theta} \\
\end{array}$} & \multirow[b]{4}{*}{ శ్ర } & $\begin{array}{l}\text { C\# } \\
1\end{array}$ & $\begin{array}{l}126 \\
0\end{array}$ & 356 & Normal & Good \\
\hline & & & & $\begin{array}{l}\text { C\# } \\
2\end{array}$ & 495 & 48 & Normal & Good \\
\hline & & & & $\begin{array}{l}\text { C\# } \\
3\end{array}$ & 990 & 61 & Normal & Good \\
\hline & & & & $\begin{array}{l}\text { C\# } \\
4 \\
\end{array}$ & 350 & 23 & Normal & Good \\
\hline \multirow{3}{*}{38} & \multirow{3}{*}{$\begin{array}{l}\text { Khashik } \\
\text { No. } 3\end{array}$} & \multirow[b]{3}{*}{ 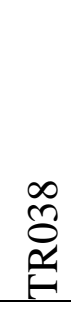 } & \multirow[b]{3}{*}{$\stackrel{\circ}{2}$} & $\begin{array}{l}\text { C\# } \\
1\end{array}$ & 495 & 68 & Normal & Good \\
\hline & & & & $\begin{array}{l}\text { C\# } \\
2 \\
\end{array}$ & 540 & 73 & Normal & Good \\
\hline & & & & $\begin{array}{l}\text { C\# } \\
3 \\
\end{array}$ & 720 & 76 & Normal & Good \\
\hline \multirow{2}{*}{39} & \multirow{2}{*}{$\begin{array}{l}\text { Khak } \\
\text { Ghariban } \\
\text { No. } 1\end{array}$} & \multirow[b]{2}{*}{ 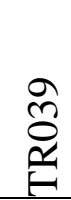 } & \multirow[b]{2}{*}{$\underset{8}{0}$} & $\begin{array}{l}\text { C\# } \\
1 \\
\end{array}$ & 540 & 200 & Normal & $\begin{array}{l}\text { Replaceabl } \\
\text { e }\end{array}$ \\
\hline & & & & $\begin{array}{l}\text { C\# } \\
2 \\
\end{array}$ & 500 & 125 & Normal & $\begin{array}{l}\text { Replaceabl } \\
\text { e }\end{array}$ \\
\hline \multirow{3}{*}{40} & \multirow{3}{*}{$\begin{array}{l}\text { Khak } \\
\text { Ghariban } \\
\text { No. } 2\end{array}$} & \multirow{3}{*}{\begin{tabular}{l} 
P \\
\multirow{0}{0}{} \\
fy
\end{tabular}} & \multirow[b]{3}{*}{8} & $\begin{array}{l}\text { C\# } \\
1 \\
\end{array}$ & 350 & 70 & Normal & $\begin{array}{l}\text { Replaceabl } \\
\text { e }\end{array}$ \\
\hline & & & & $\begin{array}{l}\text { C\# } \\
2 \\
\end{array}$ & 945 & 82 & Normal & $\begin{array}{l}\text { Replaceabl } \\
\text { e }\end{array}$ \\
\hline & & & & $\begin{array}{l}\text { C\# } \\
3\end{array}$ & 855 & 90 & Normal & $\begin{array}{l}\text { Replaceabl } \\
\text { e }\end{array}$ \\
\hline
\end{tabular}

Table (1) Status of low voltage lines of Ghazni distribution networ

\section{Discussion}

Providing high quality services, continuity of electricity supply and economical distribution network and its safety is one of the main tasks of electricity distribution companies that noncompliance with standards in the design and economic operation of distribution networks has adverse effects on all of the above in economic design and operation, waste distribution networks increase network uncertainty and waste of capital, which reduces the efficiency of the system. By observing the standards in the economic design and operation of distribution networks and waste reduction, more electricity can be supplied to the subscribers. Based on the results of this study, it is suggested to pay attention to the following: 
- Transformers should be placed in the center of gravity of the load as much as possible.

- 5 Transformers in which the voltage conversion takes place in three stages (20/6 / 0.4 $\mathrm{KV}$ ) should be replaced to transformers in which the voltage conversion takes place in two stages (20 / $0.4 \mathrm{KV}$ ).

- The length of each transformer feeder should not exceed 500 to 600 meters.

- To reduce the length of the feeders, multiple low-capacity transformers should be used instead of high-capacity transformers.

- Possibility of future development and increase of consumption according to the overload coefficient and load sharing coefficient.

- Balancing the output loads of transformers should be considered as much as possible.

- If the load of the transformer is high, replace the transformer with a transformer with a higher capacity, otherwise it will be necessary to separate the network and add another transformer to the network.

\section{Conclusion}

The studies conducted in this article show that the $0.4 \mathrm{kV}$ distribution network in Ghazni is traditionally designed and unfortunately it is not taken into consideration and is not taken seriously, and this has increased the current costs of the distribution network and in return Has and has adverse effects on consumers. Non-observance of standard distances; The choice of capacity and installation location of the transformer without technical calculations has caused voltage losses and overload of transformers in the network.

- Four transformers in the distribution network of Ghazni city are overloaded, two transformers with a capacity of $160 \mathrm{KVA}$ were $20 \%$ overloaded and two transformers with a capacity of $200 \mathrm{KVA}$ were $15 \%$ overloaded, and their overload is within the allowable limit.

- The distance of 57 circuits is in the range of (1260-700) meters, which is not allowed, and also the use of subscribers with shorter cross-section lines has reduced the voltage, which reaches 170 volts in some parts of the network. 
- The power of transformers is traditionally chosen so that one kilovolt of amperes is provided for each family.

- The electric charge graph of the Ghazni city distribution network shows that it is not a cost-effective network.

\section{Reference}

1. Factsheet," Ofgem, London, UK, Aug.2010 "Electricity Distribution Units and Loss Percentages Summary.

2. Kashem, M. A. Jasmon, G. B. and Ganapathy, V. (2000). "New approach of distribution system reconfiguration for loss minimization," International Journal of Electrical Power and Energy System, vol. 22, no. 4, pp. 269-276.

3. Lin, C.-H. (2003). "Distribution network reconfiguration for load balancing with a colored Petri net algorithm," IEE Proceedings: Communications, vol. 150, no. 3, pp. 317-324.

4. A. Dahalan, W.M.; Mokhlis, H., 2012 "Network reconfiguration for loss reduction with distributed generations using PSO," 2012 IEEE International Conference on Power and Energy (PECon), vol., no., pp.823-828.

5. C.G. Carter-Brown and C.T. Gaunt, ,2006 "Model for the apportionment of the total voltage drop in Combined Medium and Low Voltage Distribution Feeders", Journal of South African Institute of Electrical Engineers, Vol. 97(1).

6. Homaee, O. Najafi, A. Dehghanian, M. Attar, M. and Falaghi, H.( 2019).“A practical approach for distribution network load balancing by optimal rephrasing of single phase customers using discrete genetic algorithm,'International Transactions on Electrical Energy Systems, vol. 29, no. 5, pp. e2834.

7. Ukil, A. and Siti, W. (2008). "Feeder load balancing using fuzzy logic and combinatorial optimization-based implementation," Electric Power Systems Research, vol. 78, no. 11, pp. 1922-1932.

8. A. Sallam, Abdel hay, O.P. Malik (2011), Electric Distribution, System, Wiley Press. 


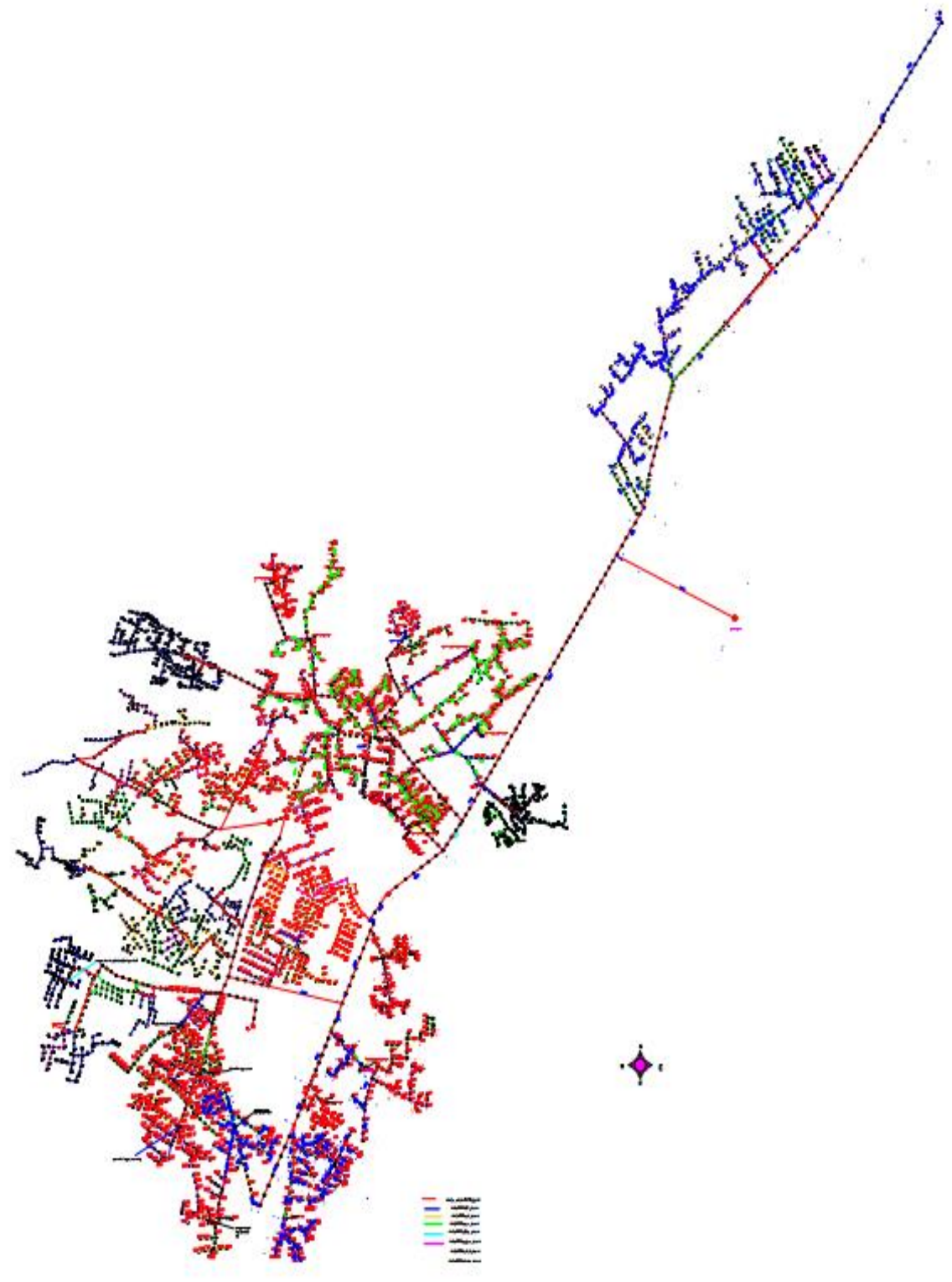

Copyright (C 2021 Massoud Danishmal, AJRSP. This is an open-access article distributed under the terms of the Creative Commons Attribution License (CC BY NC).

Doi: $\underline{\text { doi.org/10.52132/Ajrsp.e.2021.275 }}$ 